\title{
Modified frailty index as a predictor of the long-term functional result in patients undergoing primary total hip arthroplasty
}

\author{
Łukasz Pulik ${ }^{1}$, Kaja Jaśkiewicz ${ }^{2}$, Sylwia Sarzyńska ${ }^{1}$, Paweł Małdyk ${ }^{1}$, Paweł Łęgosz ${ }^{1}$ \\ ${ }^{1}$ Department of Orthopedics and Traumatology, Medical University of Warsaw, Poland \\ ${ }^{2}$ Department of Internal Medicine, Pneumology and Allergology, Medical University of Warsaw, Poland
}

\begin{abstract}
Objectives: Total hip arthroplasty (THA) is a well-approved method for the treatment of end-stage osteoarthritis (OA). Due to rising life expectancy, elderly patients burdened with multimorbidity are subjected to THA. Some of these patients present significant depletion of physiological reserves, which is described as the frailty syndrome. This study aims to assess the influence of frailty on the THA outcomes in OA patients.

Material and methods: A single-center observational study was conducted to investigate the effect of frailty measured by the modified frailty index-5 (mFl-5) and modified frailty index-11 (mFI-11) on the long-term post-THA outcomes. The analysis included 597 initially screened patients subjected to unilateral, primary THA due to hip OA. The outcomes were assessed during a follow-up visit 3 years after THA. The primary outcome measures were patient-reported (the Western Ontario and McMaster Universities Osteoarthritis Index - WOMAC) and physician-reported scales (the Harris Hip Score - HHS). The secondary outcome measures were the length of hospital stay (LOS), pain complaints, complications, and satisfaction. A correlation analysis was performed (Spearman's R). Results: Three hundred sixty-five patients met the eligibility criteria, including $57.26 \%$ women $(n=209)$ and $42.74 \%$ men $(n=156)$. The mean age was $65.11 \pm 12.12$ years. Patients with high values of mFI-5 ( $r=0.19 ; p<0.05)$ and $\mathrm{mFI}-11(r=0.22 ; p<0.01)$ achieved less satisfactory functional outcomes after THA (WOMAC). After age adjustment, mFI-11 $(r=0.17 ; p<0.05)$ was a better predictor of functional outcome (WOMAC) than $\mathrm{mFI}-5$ was $(r=0.15 ; p=0.07)$. The $\mathrm{mFI}-5(r=0.25 ; p<0.001)$ and $\mathrm{mFI}-11(r=0.29 ; p<0.001)$ correlated with longer LOS.

Conclusions: The modified frailty index-5 (mFl-5) and modified frailty index-11 (mFI-11) are useful tools to identify patients subjected to THA at a high risk of poor functioning after the procedure. They can be used in preoperative counseling before obtaining informed consent to support surgical decision-making. To our knowledge, this is the first study investigating the impact of the $\mathrm{mFI}$ on long-term postoperative functional results in OA treated with THA.
\end{abstract}

Key words: osteoarthritis, quality of life, treatment outcome, comorbidity.

\section{Introduction}

Osteoarthritis (OA) is one of the most common chronic diseases, and it is strongly linked with aging [1]. Although it is not a direct threat to the patient's life, it is one of the leading factors causing disability and depleting physical function in the older population. Furthermore, it affects overall health-related quality of life and social functioning, and causes psychological distress [2].
Total hip arthroplasty (THA) is the gold standard of treatment for the advanced stage of OA. It is acknowledged as a highly effective procedure in the reduction of pain and improvement of limb function, enabling the patient to return to everyday activities [3].

Due to the rising life expectancy and aging population, there is a continually increasing need for THA [4]. With older age, the burden of comorbidities increases, opposite to the functional reserve and resistance to external stress-

Address for correspondence:

Łukasz Pulik, Department of Orthopedics and Traumatology, Medical University of Warsaw, 61 Żwirki i Wigury St., 02-091 Warsaw,

Poland, e-mail: lukaszpulik@gmail.com

Submitted: 8.06.2020; Accepted: 5.08.2020 
ors such as surgical procedure. This state of depletion of reserves is referred to as "frailty syndrome" [5].

Patients with frailty syndrome subjected to surgical interventions have a higher incidence of complications associated with surgery and an increased mortality rate. They require prolonged hospitalization and achieve worse functional outcomes [6]. The identification of patients with frailty syndrome subjected to THA could lead to more accurate qualification for the procedure and provide high-risk patients with special perioperative care [7].

Nowadays, clinicians place particular emphasis on a holistic view of the patient, and there are useful tools available for the assessment of the clinical status of patients undergoing surgical procedures. Among them, the most common is the American Society of Anesthesiologists grades (ASA), Charlson Comorbidity Index (CCI), American College of Surgeons National Surgical Quality Improvement Program Surgical Risk Calculator (ACS NSQIP), and Elixhauser Comorbidity Method (ECM) [8]. Recently, based on ACS NSQIP data, the modified frailty index-11 (mFl-11) and modified frailty index-5 (mFl-5), were developed [9].

The authors demonstrated the high efficacy of this clinical tool in predicting mortality, postoperative complications, and prolonged hospitalization in patients subjected to THA [10]. In the study by Shin et al. [11], carried out on 14583 patients undergoing THA, mFI-11 showed better predictive properties than ASA score in complications and 30-day mortality. However, the previous research conducted on $\mathrm{mFI}-5$ and $\mathrm{mFI}-11$ has not included the aspect of the long-term physical functioning of patients, pain severity, and satisfaction rate after the THA [7].

\section{Objectives}

The study aims to investigate the influence of frailty measured with $\mathrm{mFl}-5$ and $\mathrm{mFI}-11$ on the outcomes of primary THA in OA patients. The clinical outcomes were long-term functional results (WOMAC, HHS), pain complaints (VAS), complication rate, hospitalization time (LOS), and patient's satisfaction (HKASS). Understanding the importance of frailty burden in the elderly population undergoing THA could have a substantial impact on the safety and effectiveness of this procedure.

\section{Material and methods}

\section{Trial design}

This is a single-center cohort observational study. The study received approval from the ethics committee of the Medical University of Warsaw as part of the proj- ect "Evaluation of the influence of coexisting diseases and other selected factors on the results of primary hip arthroplasty" (AKBE/196/2018).

The study followed (Strengthening the Reporting of Observational studies in Epidemiology - STROBE) guidelines for observational studies, and it was conducted according to the Guidelines of Good Clinical Practice and the principles expressed in the Declaration of Helsinki. All patients were recruited after providing written informed consent.

\section{Setting and study population}

The study was based on a retrospective analysis of clinical material from the Department of Orthopedics and Traumatology, Infant Jesus Teaching Hospital, Medical University of Warsaw. Five hundred ninety-seven patients undergoing unilateral primary THA in the five years from 2009 to 2014 were identified among the operated patients.

The exclusion criteria were: revision THA procedures, incomplete medical record, age under 18 years old, pregnancy, previous surgery of the operated joint, a diagnosis other than idiopathic OA (rheumatoid arthritis, post-traumatic osteoarthritis, developmental dysplasia of the hip, avascular necrosis, Legg-Calve-Perthes disease) or uncertain diagnosis of idiopathic OA. All patients received equivalent perioperative care.

\section{Variables and data sources}

The baseline data, including the information about comorbidities, were collected based on medical documentation in the form of medical history and operating room documentation obtained from the hospital archives and an electronic data register.

The preoperative evaluation focused on coexisting diseases and predictive factors belonging to the $\mathrm{mFI}-5$ and $\mathrm{mFI}-11$. Demographic and perioperative parameters such as age, gender, BMI, and hospitalization time were collected. Long-term results were evaluated during follow-up visits at the clinic.

During the follow-up visit, the research team collected data on the occurrence of complications after surgery, questionnaires evaluating the function of the operated limb, and the severity of pain. Long-term results of THA were evaluated with patient and physician reported outcome measures: Harris Hip Score (HHS) [12], the Western Ontario and McMaster Universities Index of Osteoarthritis (WOMAC) [13], the Visual Analogue Scale (VAS) [14], and Hip and Knee Arthroplasty Satisfaction Scale (HKASS) [15].

The WOMAC score was calculated from the Hip Osteoarthritis Outcome Score (HOOS) questionnaire ac- 
cording to the authors' recommendation [16]. During the follow-up visits, an orthopedic examination was performed. Also, information on distant postoperative complications was collected.

\section{The modified frailty index}

The Canadian Study of Health and Aging (CSHA) on the epidemiology of dementia developed the 70-item CSHA-FI based on many deficits that may be associated with frailty. The simplified version consisting of eleven health deficits was created ( $\mathrm{mFI}-11)$ because of the difficulties in measurement of each of the multiple factors and the fact that all CSHA-FI data was not uniformly collected. The $\mathrm{mFI}-11$ was proven to reflect frailty as well as CSHA-FI. Later on, $\mathrm{mFI}-11$ was simplified to the fivefactor index $\mathrm{mFI}-5$ [9]. The modified frailty index $\mathrm{mFI}-5$ and $\mathrm{mFI}-11$ can be calculated based on retrospective data from medical records. Table I explains the components of $\mathrm{mFI}-5$ and $\mathrm{mFI}-11$.

The group of patients with arterial hypertension was identified based on the diagnosis in the medical record. The use of pharmacological treatment, for this reason, was necessary for qualification. Information about the partial or total inability to function independently was obtained based on the medical history (changes in everyday activities such as problems with getting dressed, bathing, personal grooming, cooking, going out alone).

Myocardial infarction was defined as the presence of diagnosis in medical history regardless of the onset time. Peripheral vascular disease was defined as lower limb varicose veins, resting pain, presence of necrotic lesions, state after revascularization, or amputation. Impaired sensorium was defined as cognitive disorders or disturbances of consciousness. The modified frailty index-5 (mFl-5) and modified frailty index-11 (mFl-11) values are calculated by dividing the number of health deficits present by the number of health deficits measured $[9,17]$.

\section{Statistical methods}

The probability distributions of individual variables were estimated for continuous parameters. To determine the normality of the distribution of the variables, the Shapiro-Wilk test and graphical analysis were used. The analysis of relationships between continuous parameters was performed using correlation analysis with Spearman's R coefficient. The Mann-Whitney test was used to investigate the possible influence of other factors on the outcomes. The cutoff values for $\mathrm{mFI}-5$ and $\mathrm{mFI}-11$ were proposed by Dayama et al. [18]. The missing data were addressed with a complete case analysis method [19].
The results were found to be statistically significant at $p<0.05$. The statistical analysis of the collected data was performed using Statistica version 13.

\section{Results}

\section{Group characteristics}

Three hundred sixty-five patients from the initial cohort met the eligibility criteria, including $57.26 \%$ women $(n=209)$ and $42.74 \%$ men $(n=156)$. The mean age at the time of surgery was $65.11 \pm 12.12$ years (median 67 ; LQ 57; UQ 75). The mean BMI before surgery was 27.97 $\pm 5.26 \mathrm{~kg} / \mathrm{m}^{2}$ (median 27.06; LQ 24.06; UQ 30.86).

One hundred nineteen arthroplasty procedures were performed with the use of bone cement (32.60\%). The average hospitalization time among the examined patients was $16.39 \pm 5.91$ days (median 15; LQ 12; UQ 19). One hundred fifty-three patients (41.92\%) came to the follow-up visit after 1164.00 days. Clinical scales and postoperative complications were assessed in patients who completed the follow-up visit.

\section{Frailty burden}

The conditions predisposing to frailty were identified in $78.08 \%$ of patients for $\mathrm{mFI}-5$ and $80.27 \%$ for $\mathrm{mFI}-11$. The most common conditions were arterial hypertension, reported in $74.25 \%$ of patients (271), diabetes mellitus type II $19.45 \%(n=71)$, and congestive heart failure $16.44 \%(n=60)$. The prevalence of other clinical conditions belonging to $\mathrm{mFI}-5$ and $\mathrm{mFI}-11$ is presented in Table I.

The values of $\mathrm{mFI}-5$ (mean $0.26 \pm 0.19$; median 0.20; LQ 0.2; UQ 0.4) and $\mathrm{mFI}-11$ (mean $0.15 \pm 0.12$; median 0.09; LQ 0.09; UQ 0.18) were characterized by very strong positive correlation $(r=0.93 ; p<0.001)$. The distribution of $\mathrm{mFl}-5$ and $\mathrm{mFI}-11$ components in the investigated group of patients are presented in Figure 1.

\section{Functional results}

Patients with frailty ("mFI-5" and "mFI-11") had significantly less satisfactory functional results (WOMAC). The correlation index was stronger for $\mathrm{mFI}-11(r=0.22$; $p<0.05)$ than $\mathrm{mFI}-5(r=0.19 ; p<0.05)$. Also, older subjects achieved a worse clinical outcome (HHS, WOMAC).

After the age adjustment the correlation for $\mathrm{mFI}-5$ was $r=0.15, p=0.07$, and for $\mathrm{mFI}-11 r=0.17, p<0.05$. The preoperative BMI and gender did not significantly correlate with postoperative function. The results are presented in Table II.

Patients subjected to cementless THA achieved a better clinical outcome (HHS) $(p<0.01)$. Patients who experienced complications in the follow-up period had 
Table I. Prevalence of clinical conditions belonging to the modified frailty index-5 (mFl-5) and modified frailty index-11 (mFl-11)

\begin{tabular}{|lccc|}
\hline Components of clinical assessment tools & mFl-5 & mFI-11 & Prevalence \\
\hline Arterial hypertension & + & + & $74.25(271)$ \\
\hline Diabetes type I or II & + & + & $19.45(71)$ \\
\hline Congestive heart failure & + & + & $16.44(60)$ \\
\hline Partially or totally dependent & + & + & $14.52(53)$ \\
\hline Chronic obstructive pulmonary disease or pneumonia & + & + & $3.29(12)$ \\
\hline Peripheral vascular disease & & + & $9.04(33)$ \\
\hline Impaired sensorium & & + & $8.22(30)$ \\
\hline Myocardial infarction & & + & $6.85(25)$ \\
\hline $\begin{array}{l}\text { Coronary pain/cardiac surgery interventions/percutaneous } \\
\text { interventions }\end{array}$ & + & $5.75(21)$ \\
\hline Transient ischemic attack & & + \\
\hline Cerebrovascular event with neurologic deficit & + & $0.82(3)$ \\
\hline
\end{tabular}

Prevalence in \% (number of patients).

a less satisfactory clinical outcome $(H H S)(p<0.05)($ Table III).

\section{Length of stay}

There was a positive correlation between $\mathrm{mFl}-5$ and the length of stay in the hospital (LOS) $(r=0.25$; $p<0.001)$. A stronger positive correlation was confirmed for mFl-11 ( $r=0.29 ; p<0.001)$ (Fig. 2). The patients' age also correlated positively with the LOS $(r=0.12$; $p<0.05)$. The correlations were also confirmed for the age-adjusted $\mathrm{mFI}-5(r=0.22 ; p<0.001)$ and $\mathrm{mFI}-11$ $(r=0.27 ; p<0.001)$. There was no significant correlation between BMI value and the LOS. The LOS did not differ significantly by gender.

\section{Postoperative complications}

The overall incidence of complications was $18.30 \%$ $(n=28)$. The most common complication was paresthesia within the operated limb in $8.50 \%(n=13)$ of patients. Surgical site infections (SSI) rate was $4.58 \%$ $(n=7)$, while the prosthetic joint infection (PJI) incidence was $3.27 \%(n=5)$. Aseptic loosening occurred in $3.92 \%(n=6)$. The incidence of dislocation of the head

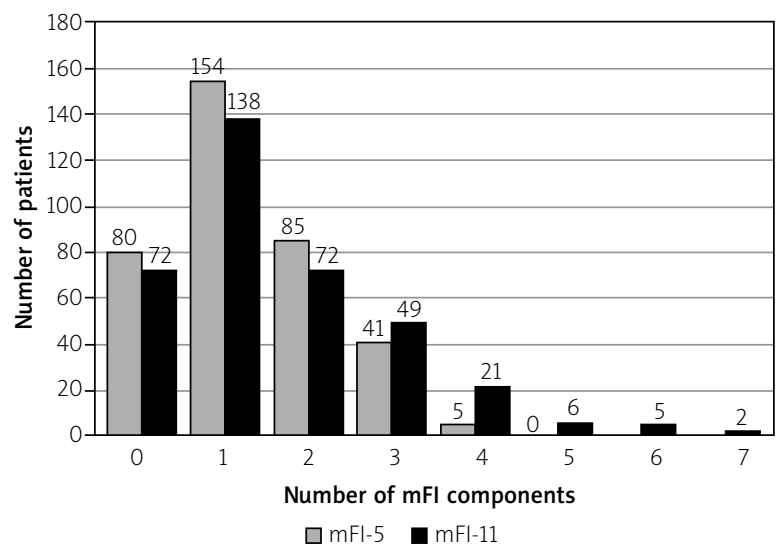

Fig. 1. The number of modified frailty index components in patients subjected to total hip arthroplasty.

of the endoprosthesis was $3.27 \%(n=5)$. There were no cases of THA periprosthetic fracture in the cohort during the observation period. Patients with $\mathrm{mFl}-5$ above the median (0.2) experienced complications more often $(15.96 \%$ vs $20.69 \% ; p>0.05)$. Similarly, patients with mFI-11 over the median (0.09) had a higher complica-

Table II. The clinical results after total hip arthroplasty

\begin{tabular}{|lcccccc|}
\hline Parameter & $\mathrm{mFl}-5$ & $\mathrm{mFI}-5 \#$ & $\mathrm{mFl}-11$ & $\mathrm{mFI}-11 \#$ & Age & BMI \\
\hline HHS & -0.12 & -0.05 & -0.15 & -0.09 & $-0.31^{\text {***}}$ & -0.14 \\
\hline VAS & 0.09 & 0.08 & 0.10 & 0.08 & 0.10 & 0.04 \\
\hline WOMAC & $0.19^{*}$ & 0.15 & $0.22^{* *}$ & $0.17^{*}$ & $0.20^{*}$ & 0.12 \\
\hline HKASS & -0.05 & -0.07 & -0.08 & -0.10 & -0.05 & -0.05 \\
\hline
\end{tabular}

HHS - Harris Hip Score, HKASS - Hip and Knee Arthroplasty Satisfaction Scale, mFI-5 - modified frailty index-5, mFl-11 - modified frailty index-11, VAS - Visual Analogue Scale, WOMAC - Western Ontario and McMaster Universities Osteoarthritis Index, Spearman's R coefficient; ${ }^{*} p<0.05,{ }^{* *} p<0.01,{ }^{* * *} p<0.001, \#$ age-adjusted. 
Table III. Patient- and physician-reported outcome measures after total hip arthroplasty

\begin{tabular}{|lcccc|}
\hline Parameter & HHS & VAS & WOMAC & HKASS \\
\hline Total & 88.40 & 4.00 & 14.00 & 91.63 \\
& $(76.85 ; 93.94)$ & $(0.00 ; 30.00)$ & $(7.00 ; 29.00)$ & $(66.64 ; 99.96)$ \\
\hline Male & 87.90 & 5.00 & 16.00 & 83.30 \\
& $(76.85 ; 92.85)$ & $(0.00 ; 32.00)$ & $(6.00 ; 29.00)$ & $(66.64 ; 99.96)$ \\
\hline Female & 88.55 & 4.00 & 14.00 & 91.63 \\
& $(77.00 ; 95.00)$ & $(0.00 ; 25.00)$ & $(7.00 ; 28.00)$ & $(66.64 ; 99.96)$ \\
\hline Cemented & $80.47^{\star *}$ & 3.50 & 18.00 & 83.30 \\
& $(70.60 ; 89.70)$ & $(0.00 ; 47.00)$ & $(7.00 ; 39.00)$ & $(66.64 ; 99.96)$ \\
\hline Not cemented & $90.00^{* *}$ & 5.00 & 12.00 & 91.63 \\
& $(79.00 ; 96.00)$ & $(0.00 ; 29.00)$ & $(5.00 ; 26.00)$ & $(58.31 ; 99.96)$ \\
\hline BMI > 25.0 & 87.00 & 5.00 & 15.00 & 91.63 \\
& $(76.85 ; 92.10)$ & $(0.00 ; 32.00)$ & $(8.00 ; 29.00)$ & $(66.64 ; 99.96)$ \\
\hline BMI < 25.0 & 91.00 & 3.50 & 11.50 & 87.47 \\
& $(77.85 ; 96.00)$ & $(0.00 ; 33.50)$ & $(4.00 ; 26.50)$ & $(66.64 ; 99.96)$ \\
\hline Any complication & $85.85^{*}$ & 4.00 & 18.00 & $66.64^{\star *}$ \\
& $(69.00 ; 91.00)$ & $(0.00 ; 47.00)$ & $(6.00 ; 41.00)$ & $(58.31 ; 91.63)$ \\
\hline No complications & $89.70^{\star}$ & & & $91.63^{* *}$ \\
& $(78.33 ; 95.00)$ & $(0.00 ; 24.50)$ & $(7.00 ; 26.00)$ & $(66.64 ; 99.96)$ \\
\hline
\end{tabular}

HHS - Harris Hip Score, VAS - Visual Analogue Scale, WOMAC - Western Ontario and McMaster Universities Osteoarthritis Index, HKASS - Hip and Knee Arthroplasty Satisfaction Scale, median (upper quartile, lower quartile), ${ }^{*} p<0.05,{ }^{* *} p<0.01,{ }^{* * *} p<0.001$.

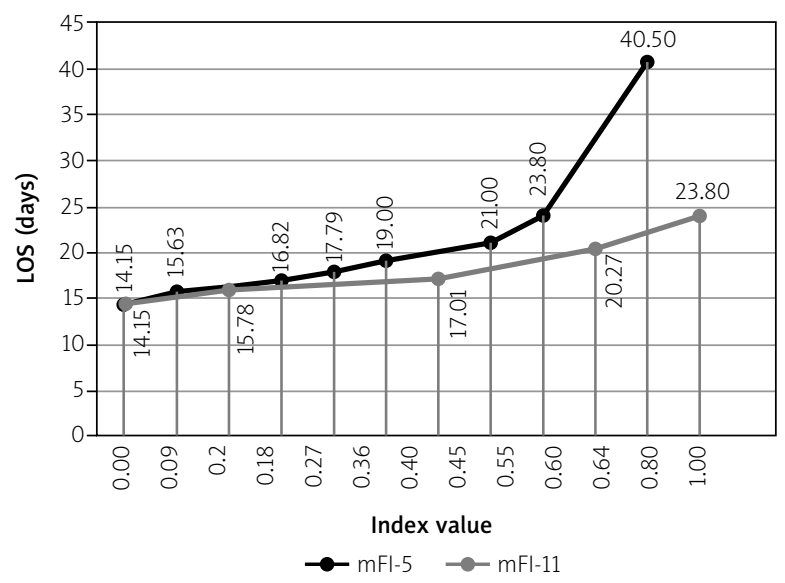

Fig. 2. Length of hospitalization of patients subjected to total hip arthroplasty and values of the modified frailty index.

tion rate $(16.67 \%$ vs $19.12 \% ; p>0.05)$. In both cases, the difference was not statistically significant. The analysis did not prove the influence of other factors on the satisfaction (HKASS) and the severity of pain (VAS) after the procedure (Table III).

\section{Discussion}

Total hip arthroplasty not only relieves pain and improves function and quality of life but may also increase the life expectancy [20]. Although it is one of the most successful surgical procedures worldwide, multiple variables may contribute to THA results. Traditional outcomes of the surgical procedures are mortality, readmission, complications, surgical recovery time, and return to work.

Nowadays, researchers highlight the importance of patient-centered outcomes such as functional status, degree of disability, emotional and mental health, and social interaction. Total hip arthroplasty results can be influenced by both patient-independent (e.g., prosthesis type, surgeon experience, hospital type) and patient-related factors (e.g., age, comorbidities, BMI, OA severity, patients' expectations, preoperative function and health-related quality of life) [21].

Although total hip arthroplasty is one of the most common orthopedic procedures in older adults, studies of the frailty and THA results are limited to the traditional outcomes [7]. In this study, we analyzed the complex impact of frailty on the outcomes of THA focusing on patient-centered outcome measures such as long-term function (WOMAC, HHS), pain sensations (VAS), and satisfaction (HKAAS). We also analyzed traditional outcomes such as surgical recovery time (LOS) and complication rate.

The systematic review of possible factors affecting short-term and long-term functional outcomes of THA indicated the negative association of high BMI, presence of comorbidities, and older age with function after the surgery. There is a positive association between factors such as physical function, mental health, and the 
functional outcome after THA. The relationships of gender, alcohol consumption, socioeconomic status, allergy, and vitamin D insufficiency and functional outcome after THA are unclear [22].

Meessen et al. [23] studied the impact of frailty (Groningen Frailty Indicator, GFI) on the functional outcomes after THA and total knee arthroplasty (TKA). In the regression analysis, the GFI had limited ability to affect the functional outcome change (HOOS) in THA patients. However, higher rates of reoperation complications and reoperation were observed in frail patients.

Schmucker et al. [7] performed a systematic review of the impact of frailty on outcomes after THA and TKA.

Only one study, by Oosting et al. [24], has assessed the functional outcome after THA (HOOS) and frailty. Although the study was mainly focused on the assessment of preoperative training on the functional in the preselected group of frail patients [7, 24]. The modified frailty index accesses mainly the patient's multimorbidity and the literature supports the influence of coexisting diseases on THA results.

In the study by Judge et al. [25] subjects with coexisting diseases achieved a less satisfactory clinical outcome during a five-year follow-up (Oxford Hip Score).

Wang et al. [26], in a multivariate regression analysis of 12 factors, confirmed the independent influence of comorbidities on the functional outcome (WOMAC). Also, Smith et al. [27] reported the negative effect of ASA status on HHS.

In our study, we proved the association between frailty and functional outcomes (WOMAC). After the age adjustment, $\mathrm{mFI}-11$ was a successful predictor of functional outcome. However, the relationship between $\mathrm{mFl}$ and functional outcomes is weak, and this indicates the complexity of factors that can affect the THA outcomes.

Okafor et al. [28] performed a systematic review of patient satisfaction and total hip arthroplasty. The studied factors included patient expectations, age, gender, pain, length of stay, and comorbidities before the surgery. Younger age is often associated with a lower satisfaction rate as patients have higher expectations and an active lifestyle. The influence of multimorbidity and comorbidities on satisfaction after THA has been studied. However, the results are inconclusive. The authors hypothesize that patients with multiple comorbidities experience a high rate of complications, and it can contribute to poor satisfaction after THA [28].

In our study, the overall satisfaction (HKASS) after THA was not affected by frailty. Understandably, patients with any THA-related complications were significantly less satisfied. The regression analysis showed that frail and pre-frail older adults in the community setting more often experience chronic pain [29].

Esses et al. [30] performed a secondary analysis of a prospective observational cohort (PRESERVE). The authors investigated frailty and its association with postsurgical pain in older patients. The frail patients were at five times higher risk of intense post-surgical pain compared to non-frail patients, but this cohort did not include THA patients [30]. We found no study on the impact of frailty on pain after the THA.

Schmucker et al. [7] suggest that frailty may impact long-term pain and function after total joint replacement. However, there is a lack of evidence for their hypothesis.

Length of stay (LOS) is one of the factors which would be considered in terms of both patients' benefits and economic burden. It is one of the most common outcome measures used to assess the patients' surgical recovery time [31]. Over the years, LOS after THA has decreased due to the dedicated protocols for rapid recovery and early discharge [32]. Patient-related factors such as comorbidities can significantly influence the LOS and hospital costs, but the evidence is limited [33].

Foote et al. [34] performed a multivariate analysis of factors influencing the LOS after THA. Older age, higher ASA, prolonged surgery time, and extensive incisions were significantly associated with delayed discharge. However, patient-independent variables can also be involved in the patients' discharge.

Husted et al. [35], in a nationwide study in Denmark, identified the logistical and clinical features of the facility to influence LOS rather than demographic factors and comorbidities. The multimodal opioid-sparing analgesia protocols, early mobilization, quick rehabilitation, and clear criteria for discharge can attribute to LOS reduction [35]. Still, emphasis should be placed on modifiable patient-related factors that can affect LOS such as high glucose and creatinine level, hypotensive events, and postoperative anemia [36]. There is evidence that frailty can also be a significant predictor of LOS.

In the study by Bellamy et al. [37], higher mFI-11 was associated with prolonged LOS after THA. Similarly, in our study, increased $\mathrm{mFI}-5$ and $\mathrm{mFI}-11$ were associated with longer LOS regardless of age. In our cohort, mean LOS was relatively long, as it was before reorganization and improvement of admitting and preparing patients for the surgery.

According to Johnson et al. [38], frailty increases the risk of wound complications, hematoma, infection, dislocations, and reoperation in THA patients. The mortality rate is also higher among frail patients [38].

Bellamy et al. [37] suggest that $\mathrm{mFI}$ is an effective risk assessment tool in the decision-making in THA pa- 
Table IV. Summary of lessons learned from the study on frailty and the total hip arthroplasty results

\begin{tabular}{|ll|}
\hline \multicolumn{1}{|c|}{ Key points } \\
\hline 1 & $\begin{array}{l}\text { THA is performed more often in elderly, multimorbid, } \\
\text { frail patients }\end{array}$ \\
\hline 2 & $\begin{array}{l}\text { Frail patients require more rigorous preoperative } \\
\text { optimization of their health status }\end{array}$ \\
\hline 3 & $\begin{array}{l}\text { An in-depth discussion to obtain informed consent } \\
\text { should always be performed }\end{array}$ \\
\hline 4 & $\begin{array}{l}\text { Frail patients are at risk of achieving less satisfactory } \\
\text { functional outcomes after THA }\end{array}$ \\
\hline 5 & $\begin{array}{l}\text { The mFI-5 and mFl-11 help to identify patients at } \\
\text { increased risk of delayed discharge }\end{array}$ \\
\hline 6 & The mFl can support the decision to qualify for THA \\
\hline THA - total hip arthroplasty, mFI-5 - modified frailty index-5, \\
mFI-11-modified frailty index-11.
\end{tabular}

tients. Patients with increased $\mathrm{mFl}-11$ had a significantly elevated risk of complication, readmission, reoperation, and mortality after THA. Also, the mFl-5 was a successful predictor for complications, readmission rate, and mortality in a retrospective analysis of patients subjected to THA [39].

However, in our study, we did not prove the increased complication risk in patients with high $\mathrm{mFl}-5$ and $\mathrm{mFl}-11$. The possible cause is that the group of patients in our study was relatively small compared to the two retrospective analyses mentioned above.

A potential bias in the study is that coexisting diseases may be underreported in medical history, especially when surgical specialties coding of diagnoses in the documentation. However, our dedicated team put particular emphasis on the quality and accuracy of the data entered [40]. Also, some inaccuracies may have arisen between the original version of $\mathrm{mFl}-5$ and $\mathrm{mFI}-11$ and the one used, due to different diagnostic criteria for specific diseases in the United States.

\section{Conclusions}

The study assessed the hypothesis that patients burdened with frailty ( $\mathrm{mFI}-5$; $\mathrm{mFI}-11$ ) subjected to THA achieve less satisfactory clinical outcomes. Analysis of the collected material confirmed the hypothesis of worse functional results in frail patients (WOMAC). After the age adjustment, the $\mathrm{mFI}-11$ was found to be a better indicator of patients' health status than $\mathrm{mFl}-5$.

Less satisfactory functional results were also observed in older patients (WOMAC, HHS). Higher frailty burden was associated with longer LOS, regardless of age ( $\mathrm{mFI}-5 ; \mathrm{mFI}-11)$.

Table IV summarizes the key points of the study. The $\mathrm{mFl}$ is a useful clinical tool, and it can be helpful in pro- spective detection of patients at risk of poor functioning after THA.

The authors declare no conflict of interest.

\section{References}

1. O'Neill TW, McCabe PS, McBeth J. Update on the epidemiology, risk factors and disease outcomes of osteoarthritis. Best Pract Res Clin Rheumatol 2018; 32: 312-326, DOI: 10.1016/j. berh.2018.10.007.

2. Salaffi F, Carotti M, Stancati A, Grassi W. Health-related quality of life in older adults with symptomatic hip and knee osteoarthritis: a comparison with matched healthy controls. Aging Clin Exp Res 2005; 17: 255-263, DOI: 10.1007/BF03324607.

3. Learmonth ID, Young C, Rorabeck C. The operation of the century: total hip replacement. Lancet 2007; 370: 1508-1519, DOI: 10.1016/S0140-6736(07)60457-7.

4. Culliford D, Maskell J, Judge A, et al. Future projections of total hip and knee arthroplasty in the UK: results from the UK Clinical Practice Research Datalink. Osteoarthritis Cartilage 2015; 23: 594-600, DOI: 10.1016/j.joca.2014.12.022.

5. Hogan DB, MacKnight C, Bergman H, et al. Models, definitions, and criteria of frailty. In: Conn's Handbook of Models for Human Aging (Second Edition) eds Ram JL, Conn PM. Academic Press 2018: 35-44.

6. Lin HS, Watts JN, Peel NM, Hubbard RE. Frailty and post-operative outcomes in older surgical patients: a systematic review. BMC Geriatr 2016; 16: 157, DOI: 10.1186/s12877-016-0329-8.

7. Schmucker AM, Hupert N, Mandl LA. The impact of frailty on short-term outcomes after elective hip and knee arthroplasty in older adults: a systematic review. Geriatr Orthop Surg Rehabil 2019; 10: 2151459319835109, DOI: 10.1177/2151459319835109.

8. Eamer G, Al-Amoodi MJH, Holroyd-Leduc J, et al. Review of risk assessment tools to predict morbidity and mortality in elderly surgical patients. Am J Surg 2018; 216: 585-594, DOI: 10.1016/j. amjsurg.2018.04.006.

9. Subramaniam S, Aalberg JJ, Soriano RP, Divino CM. New 5-Factor modified frailty index using American College of Surgeons NSQIP Data. J Am Coll Surg 2018; 226: 173-181.e8, DOI: 10.1016/j.jamcollsurg.2017.11.005.

10. Traven SA, Reeves RA, Sekar MG, et al. New 5-Factor modified frailty index predicts morbidity and mortality in primary hip and knee arthroplasty. J Arthroplasty 2019. 34: 140-144, DOI: 10.1016/j.arth.2018.09.040.

11. Shin J, Keswani A, Lovy AJ, Moucha CS. Simplified frailty index as a predictor of adverse outcomes in total hip and knee arthroplasty. J Arthroplasty 2016; 31: 2389-2394, DOI: 10.1016/j. arth.2016.04.020.

12. Harris WH. Traumatic arthritis of the hip after dislocation and acetabular fractures: treatment by mold arthroplasty. An end-result study using a new method of result evaluation. J Bone Joint Surg Am 1969; 51: 737-755.

13. Bellamy N, Buchanan WW, Goldsmith $\mathrm{CH}$, et al. Validation study of WOMAC: a health status instrument for measuring clinically important patient relevant outcomes to antirheu- 
matic drug therapy in patients with osteoarthritis of the hip or knee. J Rheumatol 1988; 15: 1833-1840.

14. Hawker GA, Mian S, Kendzerska T, French M. Measures of adult pain: Visual Analog Scale for Pain (VAS Pain), Numeric Rating Scale for Pain (NRS Pain), McGill Pain Questionnaire (MPQ), Short-Form McGill Pain Questionnaire (SF-MPQ), Chronic Pain Grade Scale (CPGS), Short Form-36 Bodily Pain Scale (SF-36 BPS), and Measure of Intermittent and Constant Osteoarthritis Pain (ICOAP). Arthritis Care Res 2011; 63: S240-S252, DOI: 10.1002/acr.20543.

15. Mahomed N, Gandhet R, Daltroy L, Katz JN. The Self-Administered Patient Satisfaction Scale for Primary Hip and Knee Arthroplasty. Arthritis 2011; 2011: 591253, DOI: 10.1155/2011/591253.

16. Pulik $九$, Romaniuk K, Jaskiewicz K, et al. An update on jointspecific outcome measures in total hip replacement. Reumatologia 2020; 58: 107-115, DOI: 10.5114/reum.2020.95366.

17. Searle SD, Mitnitski A, Gahbauer EA, et al. A standard procedure for creating a frailty index. BMC Geriatr 2008; 8: 24, DOI: 10.1186/1471-2318-8-24.

18. Dayama A, Olorunfemi O, Greenbaum S, et al. Impact of frailty on outcomes in geriatric femoral neck fracture management: an analysis of national surgical quality improvement program dataset. Int J Surg 2016; 28: 185-190, DOI: 10.1016/j. ijsu.2016.02.087.

19. Groenwold RH, White IR, Donders AR, et al. Missing covariate data in clinical research: when and when not to use the missing-indicator method for analysis. CMAJ 2012; 184: 1265-1269, DOI: 10.1503/cmaj.110977.

20. Cnudde P, Rolfson O, Timperley AJ, et al. Do patients live longer after THA and is the relative survival diagnosis-specific? Clin Orthop Relat Res 2018; 476: 1166-1175, DOI: 10.1007/ s11999.0000000000000097.

21. Hofstede SN, Gademan MG, Vliet Vlieland TP, et al. Preoperative predictors for outcomes after total hip replacement in patients with osteoarthritis: a systematic review. BMC Musculoskelet Disord 2016; 17: 212, DOI: 10.1186/s12891-016-1070-3.

22. Buirs LD, Van Beers LW, Scholtes VA, et al. Predictors of physical functioning after total hip arthroplasty: a systematic review. BMJ Open 2016; 6: e010725, DOI: 10.1136/bmjopen-2015-010725.

23. Meessen JMTA, Fiocco M, Leichtenberg CS, et al. Frailty questionnaire is not a strong prognostic factor for functional outcomes in hip or knee arthroplasty patients. Geriatr Orthop Surg Rehabil 2019; 10: 2151459318808164, DOI: 10.1177/2151459318808164.

24. Oosting E, Jans MP, Dronkers JJ, et al. Preoperative home-based physical therapy versus usual care to improve functional health of frail older adults scheduled for elective total hip arthroplasty: a pilot randomized controlled trial. Arch Phys Med Rehabil 2012; 93: 610-616, DOI: 10.1016/j.apmr.2011.11.006.

25. Judge A, Arden NK, Batra RN, et al. The association of patient characteristics and surgical variables on symptoms of pain and function over 5 years following primary hip-replacement surgery: a prospective cohort study. BMJ Open 2013; 3: e002453, DOI: 10.1136/bmjopen-2012-002453.
26. Wang W, Morrison TA, Geller JA, et al. Predicting short-term outcome of primary total hip arthroplasty: a prospective multivariate regression analysis of 12 independent factors. J Arthroplasty 2010; 25: 858-864, DOI: 10.1016/j.arth.2009.06.011.

27. Smith GH, Johnson S, Ballantyne JA, et al. Predictors of excellent early outcome after total hip arthroplasty. J Orthop Surg Res 2012; 7: 13, DOI: 10.1186/1749-799X-7-13.

28. Okafor L, Chen AF. Patient satisfaction and total hip arthroplasty: a review. Arthroplasty 2019; 1: 6, DOI: 10.1186/ s42836-019-0007-3.

29. Nakai Y, Makizako H, Kiyama R, et al. Association between chronic pain and physical frailty in community-dwelling older adults. Int J Environ Res Public Health 2019; 16: 1330, DOI: 10.3390/ijerph16081330.

30. Esses GJ, Liu X, Lin HM, et al. Preoperative frailty and its association with postsurgical pain in an older patient cohort. Reg Anesth Pain Med 2019; 44: 695-699, DOI: 10.1136/rapm2018-100247.

31. Bergman S, Feldman LS, Barkun JS. Evaluating surgical outcomes. Surg Clin North Am 2006; 86: 129-149, DOI: 10.1016/j. suc.2005.10.007.

32. Stambough JB, Nunley RM, Curry MC, et al. Rapid recovery protocols for primary total hip arthroplasty can safely reduce length of stay without increasing readmissions. J Arthroplasty 2015; 30: 521-526, DOI: 10.1016/j.arth.2015.01.023.

33. Olthof $M$, Stevens $M$, Bulstra SK et al. The association between comorbidity and length of hospital stay and costs in total hip arthroplasty patients: a systematic review. J Arthroplasty 2014; 29: 1009-1014, DOI: 10.1016/j.arth.2013.10.008.

34. Foote J, Panchoo K, Blair P, Bannister G. Length of stay following primary total hip replacement. Ann R Coll Surg Engl 2009; 91: 500-504, DOI: 10.1308/003588409X432356.

35. Husted H, Hansen HC, Holm G, et al. What determines length of stay after total hip and knee arthroplasty? A nationwide study in Denmark. Arch Orthop Trauma Surg 2009; 130: 263268, DOI: 10.1007/s00402-009-0940-7.

36. Farley KX, Anastasio AT, Premkumar A, et al. The influence of modifiable, postoperative patient variables on the length of stay after total hip arthroplasty. J Arthroplasty 2019; 34: 901906, DOI: 10.1016/j.arth.2018.12.041.

37. Bellamy JL, Runner RP, Vu CCL, et al. Modified Frailty index is an effective risk assessment tool in primary total hip arthroplasty. J Arthroplasty 2017; 32: 2963-2968, DOI: 10.1016/j. arth.2017.04.056.

38. Johnson RL, Abdel MP, Frank RD, et al. Impact of frailty on outcomes after primary and revision total hip arthroplasty. J Arthroplasty 2019; 34: 56-64.e5, DOI: 10.1016/j. arth.2018.09.078.

39. Traven SA, Reeves RA, Sekar MG, et al. New 5-Factor modified Frailty index predicts morbidity and mortality in primary hip and knee arthroplasty. J Arthroplasty 2019; 34: 140-144, DOI: 10.1016/j.arth.2018.09.040.

40. Quan H, Parsons GA, Ghali WA. Validity of information on comorbidity derived rom ICD-9-CCM administrative data. Med Care 2002; 40: 675-685, DOI: 10.1097/00005650200208000-00007. 